\title{
Immunization of traffic-driven epidemic spreading
}

\author{
Han-Xin Yang荬 and Bing-Hong Wang ${ }^{2}$ \\ ${ }^{1}$ Department of Physics, Fuzhou University, Fuzhou 350108, China \\ ${ }^{2}$ Department of Modern Physics, University of Science and Technology of China, Hefei, 230026, China
}

\begin{abstract}
In this paper, we study the control of the traffic-driven epidemic spreading by immunization strategy. We consider the random, degree-based and betweeness-based immunization strategies, respectively. It is found that the betweeness-based immunization strategy can most effectively prevent the outbreak of traffic-driven epidemic. Besides, we find that the critical number of immune nodes above which epidemic dies out is increased with the enhancement of the spreading rate and the packet-generation rate.
\end{abstract}

Keywords: traffic-driven epidemic spreading; immunization strategy; betweeness

\section{INTRODUCTION}

Both epidemic spreading [1-11] and traffic transportation [12-18] on complex networks have attracted much attention in the past decade. For a long time, the two types of dynamical processes have been studied independently. The first attempt to incorporate traffic into epidemic spreading is based on metapopulation model [24-29]. This framework describes a set of spatially structured interacting subpopulations as a network, whose links denote the traveling path of individuals across different subpopulations. Each subpopulation consists of a large number of individuals. An infected individual can infect other individuals in the same subpopulation. The metapopulation model is often used to simulate the spread of human and animal diseases (such as SARS and H1N1) among different cities. In a recent work, Meloni et al. proposed another traffic-driven epidemic spreading model [30], in which each node of a network represents a router in the Internet and the epidemic can spread between nodes by the transmission of packets. A susceptible node will be infected with some probability every time it receives a packet from an infected neighboring node. Meloni model can be applied to study the propagation of computer virus in the Internet.

Meloni model has received increasing attention in recent years. It has been found that the routing strategy plays an important role in Meloni model. Epidemic spreading can be effectively controlled by a local routing strategy [32] or an efficient routing protocol [33]. For a given routing strategy, epidemic spreading is affected by network structures. The increase of the average network connectivity can slow down the epidemic outbreak [34]. Besides, the epidemic threshold can be enhanced by the targeted cutting of links among large-degree nodes or edges with the largest algorithmic betweenness [35].

Previous studies have shown that immunization is an effective way to inhibit traditional epidemic spreading in which infections are transmitted as a reaction process from nodes to all neighbors [36, 37]. In this paper, we will study how different immunization strategies affect the traffic-driven epidemic spreading in Meloni model. Three immunization strategies: the random immunization, the targeted immunization of nodes with the largest degree and the targeted immunization of nodes with the largest algorithmic betweenness are considered, respectively. It is found that, the targeted immunization of nodes with the largest algorithmic betweenness can most effectively inhibit the traffic-driven epidemic spreading.

\section{TRAFFIC-DRIVEN EPIDEMIC SPREADING MODEL AND IMMUNIZATION STRATEGIES}

Following the work of Meloni et al. [30], we incorporate the traffic dynamics into the classical susceptible-infectedsusceptible model [38] of epidemic spreading as follows. In a network of size $N$, at each time step, $\lambda N$ new packets are generated with randomly chosen sources and destinations (we call $\lambda$ as the packet-generation rate), and each node can deliver at most $C$ packets towards their destinations. Packets are forwarded according to a given routing algorithm. The queue length of each agent is assumed to be unlimited. The first-in-first-out principle applies to the queue. Each newly generated packet is placed at the end of the queue of its source node. Once a packet reaches its destination, it is removed from the system. After a transient time, the total number of delivered packets at each time will reach a steady value, then an initial fraction of nodes $\rho_{0}$ is set to be infected (we set $\rho_{0}=0.1$ in numerical experiments). The infection spreads in the network through packet exchanges. Each susceptible node has the probability $\beta$ of being infected every time it receives a packet from an infected neighbor. The infected nodes recover at rate $\mu$ (we set $\mu=1$ in this paper).

Once a node becomes immune, it cannot be infected and thus does not transmit the infection to their neighbors. We consider three immunization strategies respectively. (I) The random strategy (RS): we randomly set $n$ nodes to be immunized from the

\footnotetext{
*Electronic address: hxyang01@gmail.com
} 


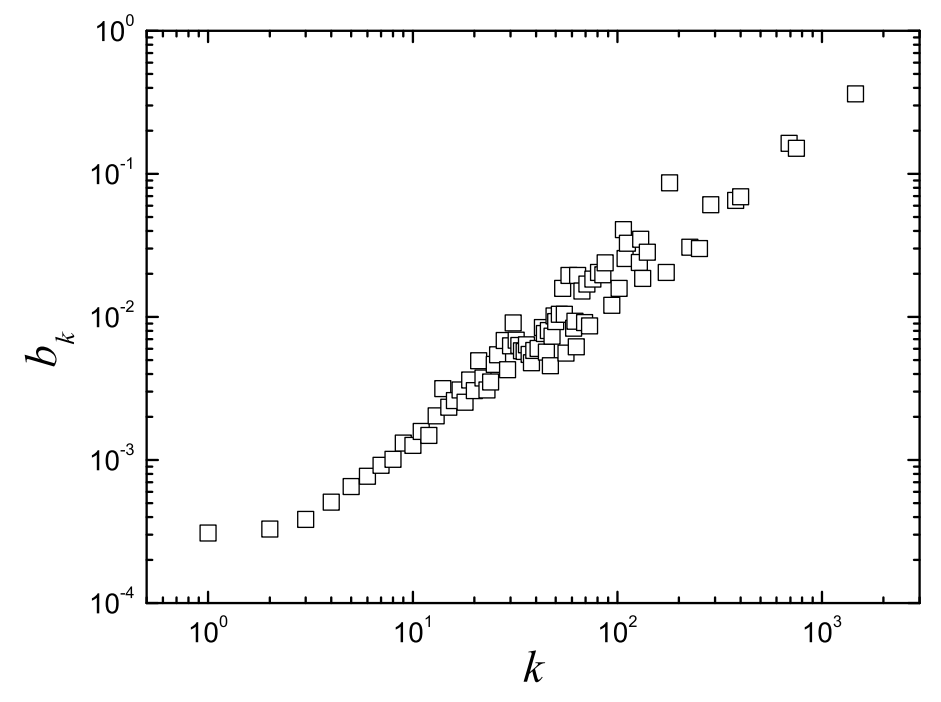

FIG. 1: The dependence of the algorithmic betweenness $b_{k}$ on degree $k$.

network. (II) The degree-based strategy (DS): we select $n$ nodes with the largest degree to be immunized. (III) The betweennessbased strategy (BS): we choose $n$ nodes with the largest algorithmic betweenness to be immunized.

\section{RESULTS AND DISCUSSIONS}

In the following, we carry out simulations systematically by employing traffic-driven epidemic spreading on top of the Internet maps at the autonomous system level [39], where the network size $N=6474$, the average degree $\langle k\rangle=3.88$, and the degree distribution follows a power law form $P(k) \sim k^{-\gamma}$ with $\gamma \approx 2.2$. Without special mention, we use the the shortest-path routing algorithm to deliver packets. Moreover, we assume that the node-delivering capacity $C$ is infinite, so that traffic congestion will not occur in the network.

The algorithmic betweenness of a node $k$ is defined as [40]:

$$
b_{k}=\frac{1}{N(N-1)} \sum_{i \neq j} \frac{\sigma_{i j}(k)}{\sigma_{i j}},
$$

where $\sigma_{i j}$ is the total number of possible paths going from $i$ to $j$ according to a specific routing algorithm, $\sigma_{i j}(k)$ is the number of such paths running through node $k$, and the sum runs over all pairs of nodes. The algorithmic betweenness of a node represents the average number of packets passing through that node at each time step when the packet-generation rate $\lambda=1 / N$.

Figure 1 shows the dependence of the algorithmic betweenness $b_{k}$ on degree $k$. A general trend is that, $b_{k}$ increases with $k$, and the relationship between $b_{k}$ and $k$ approximatively follows a power-law form as $b_{k} \sim k^{\nu}$. We need to point out that, a larger-degree node may have lower algorithmic betweenness. For example, $b_{k}$ is $8.7 \times 10^{-3}$ for $k=73$, which is smaller than that for $k=58$, whose algorithmic betweenness is $2.0 \times 10^{-2}$.

Figure 2 shows the density of infected nodes $\rho$ as a function of the spreading rate $\beta$ in RS, DS, and BS cases. One can observe that for each case, there exists an epidemic threshold $\beta_{c}$, beyond which the density of infected nodes is nonzero and increases as $\beta$ is increased. For $\beta<\beta_{c}$, the epidemic goes extinct and $\rho=0$.

Figure 3 shows the epidemic threshold $\beta_{c}$ as a function of the number of immune nodes $n$ in RS, DS, and BS cases. For RS case, the epidemic threshold $\beta_{c}$ is almost unchanged when the number of immune nodes is small, indicating that the random immunization is useless. For DS and BS cases, $\beta_{c}$ increases as $n$ increases, indicating that more immune nodes can better suppress the outbreak of epidemic. Moreover, from Fig. 3 one can find that for the same value of $n, \beta_{c}$ is the highest in BS case. For BS case, only ninety immune nodes (about $1.4 \%$ nodes) can completely prevent the outbreak of epidemic even the spreading rate is 1 . The above phenomena manifest that, $\mathrm{BS}$ is the most effective immunization strategy in the traffic-driven epidemic spreading.

Figure 4 shows the density of infected nodes $\rho$ as a function of the number of immune nodes $n$ in DS and BS cases. One can observe that there exists a critical number of immune nodes $n_{c}$, above which the epidemic goes extinct and $\rho=0$. Undoubtedly, the smaller $n_{c}$ reduces the cost of immunization. Figure 5 (a) shows that $n_{c}$ as a function of the spreading rate $\beta$ in DS and BS 


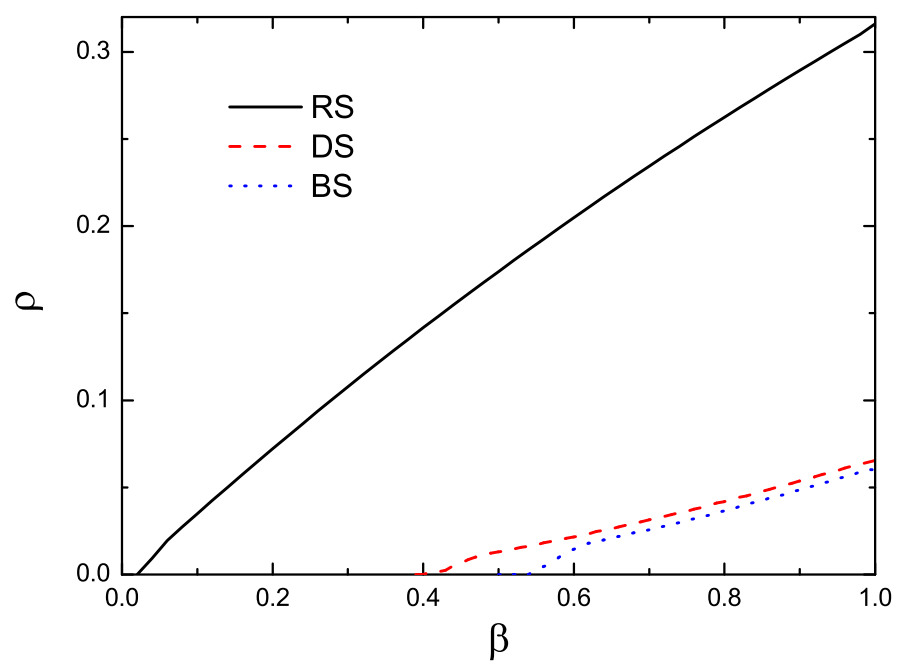

FIG. 2: (Color online) Density of infected nodes $\rho$ as a function of the spreading rate $\beta$ in RS, DS, and BS cases. The packet-generation rate $\lambda=0.3$ and the number of immune nodes $n=50$. Each curve is an average of 100 different realizations.

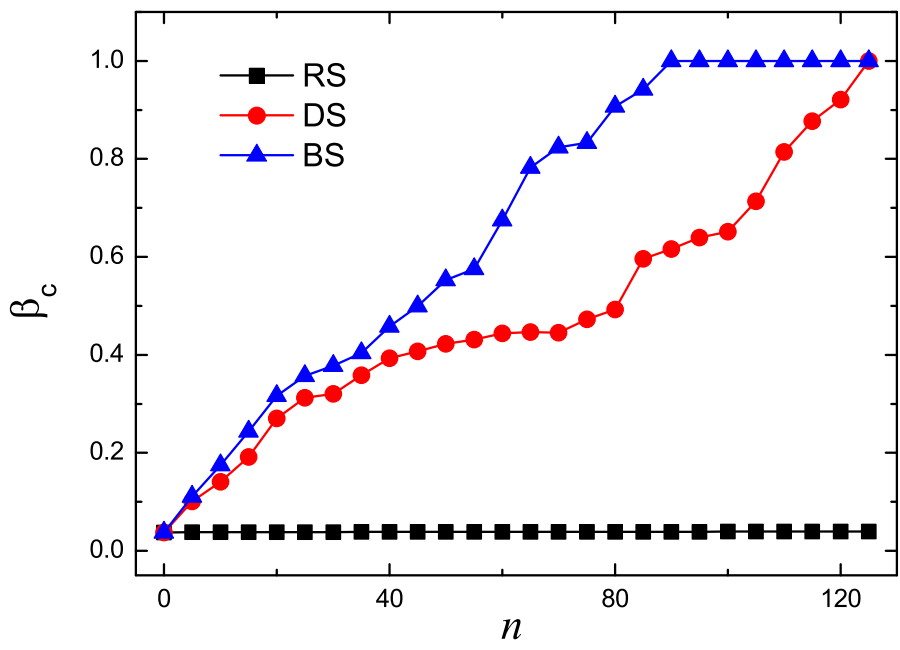

FIG. 3: (Color online) The epidemic threshold $\beta_{c}$ as a function of the number of immune nodes $n$ in DS and BS cases. The packet-generation rate $\lambda=0.3$. Each data point results from an average over 100 different realizations.

cases. One can see that $n_{c}$ increases with $\beta$. Besides, for the same value of $\beta, n_{c}$ is smaller in the case of BS than that of DS. Figure 5 (b) shows that $n_{c}$ as a function of the packet-generation rate $\lambda$ in DS and BS cases. One can see that $n_{c}$ increases with $\lambda$, manifesting that the increase of traffic flow is unfavorable for the control of epidemic spreading. For the same value of $n, n_{c}$ is smaller in the case of BS than that of DS. From Fig. 5, one can find that, compared to the degree-based immunization strategy, the betweenness-based immunization strategy is more effective in suppressing the traffic-driven epidemic spreading.

In all the above studies, packets are forwarded following the shortest-path routing algorithm. In fact, our finding that betweenness-based immunization strategy can most effectively prevent the outbreak of traffic-driven epidemic is robust with respect to different kinds of routing algorithms. Figure 6 shows the epidemic threshold $\beta_{c}$ as a function of the number of immune nodes $n$ in RS, DS, and BS cases when the efficient routing algorithm is applied. The efficient routing algorithm is described as follows [41]. For any path between nodes $i$ and $j, P(i \rightarrow j):=i \equiv x_{1}, x_{2}, \cdots, x_{n} \equiv j$, we define

$$
L(P(i \rightarrow j): \alpha)=\sum_{l=1}^{n} k\left(x_{l}\right)^{\alpha},
$$

where $k\left(x_{l}\right)$ is the degree of node $x_{l}$ and $\alpha$ is a tunable parameter. For any given $\alpha$, the efficient path between $i$ and $j$ is corresponding to the route that makes the sum $L(P(i \rightarrow j): \alpha)$ minimum. In this paper, we set the routing parameter $\alpha=1$. 


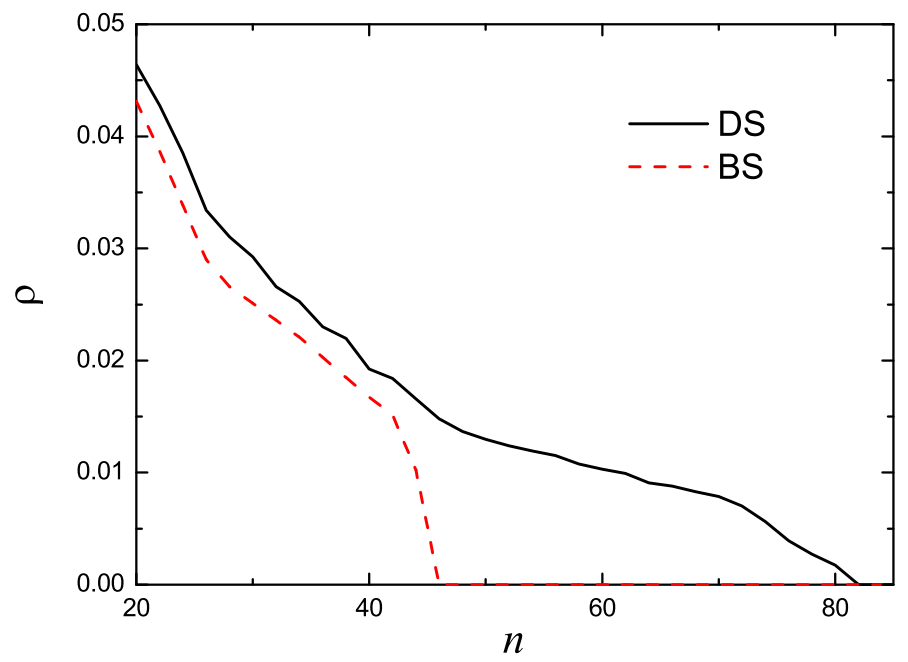

FIG. 4: (Color online) The density of infected nodes $\rho$ as a function of the number of immune nodes $n$ in DS and BS cases. The packetgeneration rate $\lambda=0.3$ and the spreading rate $\beta=0.5$. Each curve results from an average over 100 different realizations.
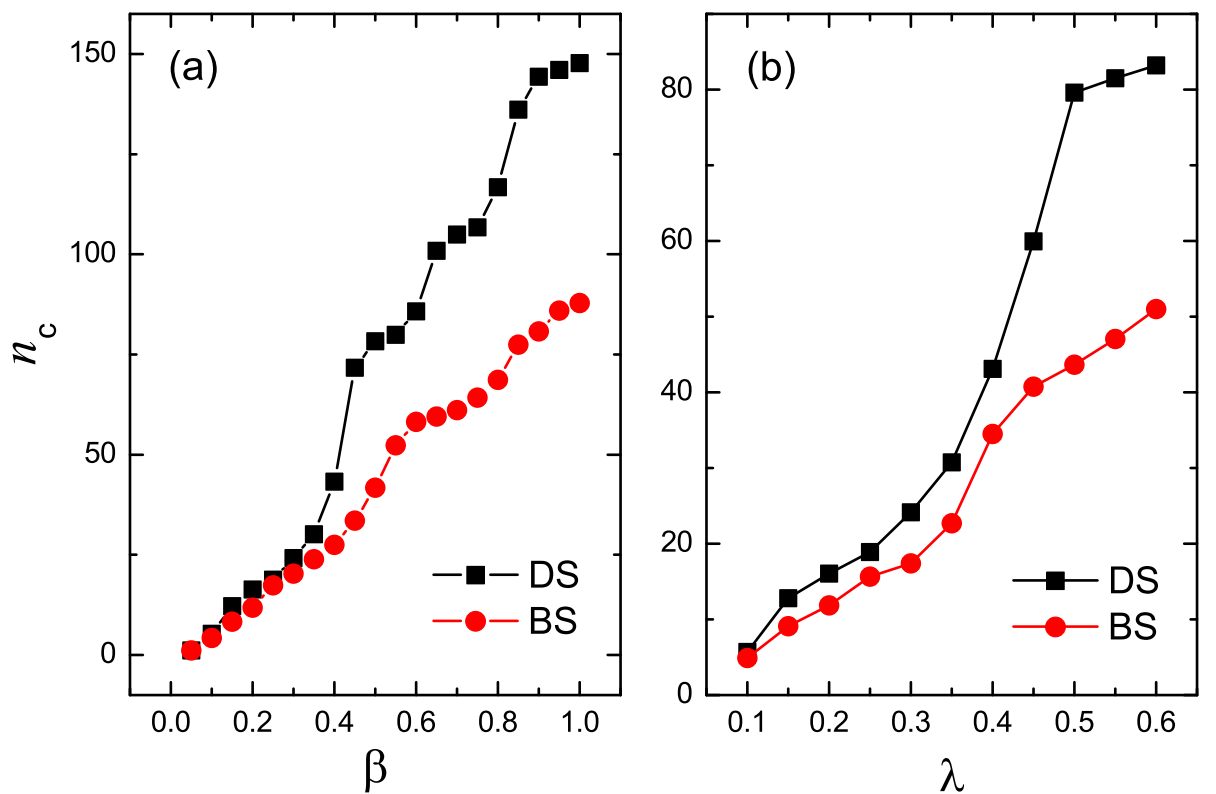

FIG. 5: (Color online) (a) The critical number of immune nodes $n_{c}$ as a function of the spreading rate $\beta$ in DS and BS cases. The packetgeneration rate $\lambda=0.3$. (b) The critical number of immune nodes $n_{c}$ as a function of the packet-generation rate $\lambda$ in DS and BS cases. The spreading rate $\beta=0.3$. Each data point results from an average over 100 different realizations.

From Fig. 6, one can find that for the same number of immune nodes $n, \beta_{c}$ is the largest in BS case while $\beta_{c}$ keeps almost unchanged in RS case.

\section{CONCLUSION}

In conclusion, we have studied the effects of immunization strategies on traffic-driven epidemic spreading. Our results show that, the outbreak of traffic-driven epidemic can be effectively suppressed when a small fraction of nodes with the largest algorithmic betweenness are immunized. This finding is robust with respect to different kinds of routing algorithms including the shortest-path routing algorithm and the efficient routing algorithm. For traditional epidemic spreading where infections are 


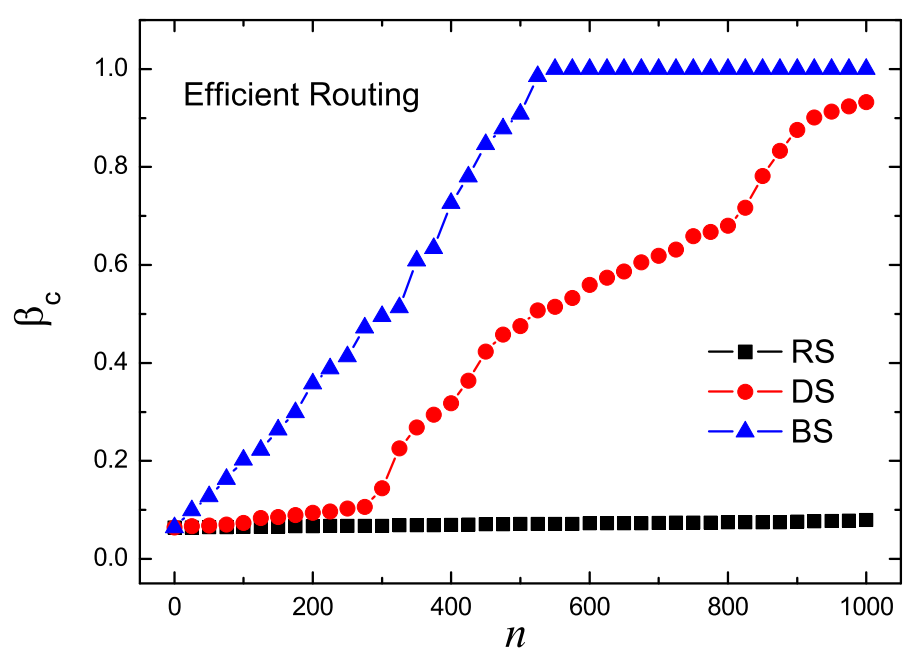

FIG. 6: (Color online) The epidemic threshold $\beta_{c}$ as a function of the number of immune nodes $n$ in DS and BS cases when the efficient routing algorithm is applied. The packet-generation rate $\lambda=0.3$. Each data point results from an average over 100 different realizations.

transmitted as a reaction process from nodes to all neighbors, an effective immunization strategy is to vaccinate the largest-degree nodes [36]. However, compared to the betweenness-based immunization strategy, the degree-based immunization strategy is less efficient in the suppression of traffic-driven epidemic spreading. This is because the larger-degree nodes may not have higher algorithmic betweenness. Moreover, we find that more immune nodes are needed to prevent the outbreak of epidemic as the spreading rate and the packet-generation rate are increased. We hope our results can be useful to control traffic-driven epidemic spreading.

\section{Acknowledgments}

This work was supported by the National Science Foundation of China (Grant Nos. 61403083, 11275186, 91024026 and 71301028), and the Natural Science Foundation of Fujian Province, China (Grant No. 2013J05007).

[1] R. Pastor-Satorras, A. Vespignani, Phys. Rev. Lett. 86 (2001) 3200.

[2] M. E. J. Newman, Phys. Rev. E 66 (2002) 016128.

[3] M. Barthélemy, A. Barrat, R. Pastor-Satorras, A. Vespignani, Phys. Rev. Lett. 92 (2004) 178701

[4] G. Yan, Z.-Q. Fu, J. Ren, W.-X. Wang, Phys. Rev. E 75 (2007) 016108.

[5] M. Kitsak, L. K. Gallos, S. Havlin, F. Lijeros, L. Muchnik L, H. E. Stanley, H. A. Makse, Nat. Phys. 6 (2010) 888.

[6] R. Parshani, S. Carmi, S. Havlin, Phys. Rev. Lett. 104 (2010) 258701.

[7] C. Castellano, R. Pastor-Satorras, Phys. Rev. Lett. 105 (2010) 218701.

[8] B. Karrer, M. E. J. Newman, Phys. Rev. E 84 (2011) 036106.

[9] C. Castellano, R. Pastor-Satorras, Sci. Rep. 2 (2012) 372.

[10] M. Dickison, S. Havlin, H. E. Stanley, Phys. Rev. E 85 (2012) 066109.

[11] W. Wang, M. Tang, H. Yang, Y.-H. Do, Y.-C. Lai, G.-W. Lee, Sci. Rep. 4 (2014) 5097.

[12] J. Shang, L. Liu, X. Li, F. Xie, C. Wu, Physica A 419 (2015) 171.

[13] P. Echenique, J. Gómez-Gardeñes, Y. Moreno, Phys. Rev. E 70 (2004) 056105.

[14] W.-X. Wang, B.-H. Wang, C.-Y. Yin, Y.-B. Xie, T. Zhou, Phys. Rev. E 73 (2006) 026111.

[15] W.-X. Wang, C.-Y. Yin, G. Yan, B.-H. Wang, Phys. Rev. E 74 (2006) 016101.

[16] S. Meloni, J. Gómez-Gardeñes, V. Latora, Y. Moreno, Phys. Rev. Lett. 100 (2008) 208701.

[17] Z.-X. Wu, W.-X. Wang, K.-H. Yeung, New J. Phys 10 (2008) 023025.

[18] M. Tang, Z. Liu, X. Liang, P. M. Hui, Phys. Rev. E 80 (2009) 026114.

[19] H.-X. Yang, W.-X. Wang, Y.-B. Xie, Y.-C. Lai, B.-H. Wang, Phys. Rev. E 83 (2011) 016102.

[20] W.-B. Du, Z.-X. Wu, K.-Q. Cai, Physica A 392 (2013) 3505.

[21] W. Huang, X. Yang, X. Yang, S. Chen, Physica A 410 (2014) 22.

[22] H.-X. Yang, M. Tang, Physica A 402 (2014) 1. 
[23] C. Liu, W.-B. Du, W.-X. Wang, PLoS ONE 9 (2014) e97822.

[24] V. Colizza, A. Barrat, M. Barthélemy, A. Vespignani, Proc. Natl Acad. Sci. USA 103 (2006) 2015.

[25] V. Colizza, A. Vespignani, Phys. Rev. Lett. 99 (2007) 148701.

[26] M. Tang, L. Liu, Z. Liu, Phys. Rev. E 79 (2009) 016108.

[27] D. Balcan, A. Vespignani, Nat. Phys. 7 (2011) 581.

[28] Z. Ruan, P. Hui, H. Lin, Z. Liu, Eur. Phys. J. B 86 (2013) 13.

[29] Y.-W. Gong, Y.-R. Song, G.-P. Jiang, Physica A 416 (2014) 208.

[30] S. Meloni, A. Arena, Y. Moreno, Proc. Natl Acad. Sci. USA 106 (2009) 16897.

[31] S. Meloni, N. Perra, A. Arenas, S. Gómez, Y. Moreno, A. Vespignani, Sci. Rep. 1 (2011) 62.

[32] H.-X. Yang, W.-X. Wang, Y.-C. Lai, Y.-B. Xie, B.-H. Wang, Phys. Rev. E 84 (2011) 045101.

[33] H.-X. Yang, Z.-X. Wu, J. Stat. Mech. (2014) P03018

[34] H.-X. Yang, W.-X. Wang, Y.-C. Lai, Chaos 22 (2012) 043146.

[35] H.-X. Yang, Z.-X. Wu, B.-H. Wang, Phys. Rev. E 87 (2013) 064801.

[36] R. Pastor-Satorras, A. Vespignani, Phys. Rev. E 65 (2002) 036104.

[37] Q. Wu, X. Fu, Z. Jin, M. Small, Physica A 419 (2015) 566.

[38] N. T. J. Bailey, The Mathematical Theory of Infectious Diseases, Griffin, London, 1975.

[39] The data is available from http://snap.stanford.edu/data/

[40] A. Arenas, A. Díaz-Guilera, R. Guimerà, Phys. Rev. Lett. 86 (2001) 3196.

[41] G. Yan, T. Zhou, B. Hu, Z.-Q. Fu, and B.-H. Wang, Phys. Rev. E 73 (2006) 046108. 